\title{
La migración de retorno y la inserción integral al contexto escolar y comunitario: propuesta de una política a beneficio de la niñez y adolescencia-familias migrantes en Honduras
}

\section{Dora Suyapa Díaz Quintero ${ }^{1}$ - Diana Claudeth Sabillón Zelaya²}

Recepción: 02-11-2020 / Aceptación: 28-01-21

\section{Resumen}

El presente artículo aborda la realidad de la niñez y juventud migrante de retorno e integración al aula escolar en el contexto escolar y comunitario en las zonas de alto flujo migratorio en Honduras. El objetivo del estudio fue analizar el fenómeno de la experiencia migratoria en retorno de niños y adolescentes en el contexto hondureño, en los departamentos de Francisco Morazán, Yoro, Olancho y Cortés; los cuales son representativos en alta convergencia poblacional al flujo migratorio de retorno, y a la participación en procesos de inserción al aula escolar en Honduras.

La investigación se realizó a partir del enfoque cualitativo, en la cual se utilizaron elementos de tipo etnográfico y revisión de documentos, mediante el uso de la teoría fundamentada a través de las técnicas: entrevistas semiestructuradas y grupos focales a una muestra intencionada de 67 niños y adolescentes; 31 docentes y 65 padres y tutores. Uno de los principales hallazgos consistió en determinar que, el retorno e inserción de niños y adolescentes en Honduras no posee una inserción integral, por tanto, se requiere de una educación reconstructiva, asequible mediante procesos de adecuación curricular y la capacitación a docentes y padres de familia.

Palabras clave: Niñez y juventud migrante de retorno, inserción integral, inserción escolar y comunitaria, Política migratoria educativa.

\begin{abstract}
This article addresses the reality of return migrant children and youth and integration into the school classroom and community context in areas of high migratory flow in Honduras. The objective of the study was to analyze the phenomenon of the migratory experience in the return of children and adolescents in the Honduran context in the departments of Francisco Morazán, Yoro, Olancho and Cortés, which are representative in high population convergence to the return migratory flow and to participation in processes of integration into the school classroom in Honduras.

The research was carried out from the qualitative approach, in which ethnographic elements and document review were used, through the use of theory based in the following techniques: semi-structured interviews and focus groups to an intentional sample of 67 children and teenagers; 31 teachers and 65 parents and guardians. One of the main findings consisted in determining that the return and insertion of children and adolescents in Honduras does not have a comprehensive integration; therefore, it requires a reconstructive education, affordable through curricular adaptation processes and teachers and parents training.
\end{abstract}

Key words: Return migrant children and youth, comprehensive integration, school and community integration, Migration education policy.

1. Doctora en Ciencias Sociales en Gestión del Desarrollo Humano, jefa de la Unidad de Investigación y Pedagogía Universidad Nacional Autónoma de Honduras; e-mail: dora.diaz@unah.edu.hn; ORCID: https://orcid.org/0000-0001-5080-7337

2. Máster en Ciencias de la Educación: Mención Didáctica e Innovación Pedagógica, Docente auxiliar, Universidad Nacional Autónoma de Honduras; e-mail: diana.sabillon@unah.edu.hn; ORCID: https://orcid.org/0000-0002-9366-4222 


\section{Introducción}

Los alcances de esta investigación, su título y línea versan sobre la "Movilidad y Migración”, con el fin de impulsar políticas educativas para la migración, políticas de protección social y suscitar la asistencia social; así como la seguridad social en los niños y adolescentes $(\mathrm{NA})^{3}$, la atención de la población urbana y rural en condición de pobreza; pobreza extrema, vulnerabilidad, riesgo y exclusión social. En este contexto hay que señalar que, en las últimas décadas, a nivel internacional $-y$ en particular la región centroamericana- ha experimentado importantes flujos migratorios de NA. Según Organización Internacional para las Migraciones-OIM (2015, p.10), alrededor del $80 \%$ de los adolescentes entre 12 y 14 años de edad migran con sus padres; mientras que quienes están entre los 15 y 17 años lo hacen en un 50\%, y solo el $20 \%$ de estos movimientos corresponde a quienes oscilan entre los 18 y 24 años.

Dichos procesos migratorios se ven inducidos por problemas en la economía familiar, la pobreza, la inseguridad ciudadana, amenazas por parte de organización de grupos ilícitos, falta de acceso a la educación, falta de empleo, entre otros. De acuerdo con este estudio, los padres de familias migrantes han indicado que las causas principales para salir del país son mejorar las condiciones de vida (55\%), buscar trabajo (33\%) y resolver problemas familiares (12\%) (ver figura 1).
Por estas razones, en Honduras se ha generado un nuevo flujo migratorio de niños y jóvenes bajo la modalidad de "caravana", el cual tuvo un fuerte auge desde el año 2018 hasta inicios del 2020. Esta situación ha modificado los patrones de migración y acrecentado las cifras de NA acompañados y no acompañados; según Santos (2020, pág.16), estas caravanas se transformarán en un movimiento social $y$, por tanto, aumentará la vulnerabilidad de este rubro.

\section{Emigración infantil y políticas públicas}

Para las políticas públicas y leyes, la emigración infantil constituye un proceso de reagrupación familiar, desde los países de origen hacia los de destino. Para Gaitán (2008), citado por Pivez Soto (2011, p.16), “el concepto de reagrupación familiar implica enfocar la mirada desde la sociedad de destino principalmente (etnocentrismo), porque se presupone que es allí donde la familia se reunirá nuevamente en virtud de una visión lineal del proceso migratorio". Esta es una de las aspiraciones de movilidad migratoria por la cual los padres de familia toman la decisión de migrar junto a sus hijos, manteniendo la familia unida y con el ideal de una inserción escolar en el país de emigración; o en el caso de un retorno a Honduras, en una reinserción escolar exitosa.

La educación pública que se ofrece en los diversos países ha mostrado que como instituciones escolares han transformado y ade-

3. Dentro del contexto de la lectura del artículo, las autoras se referirán a los sujetos de estudio mediante el acrónimo NA. 


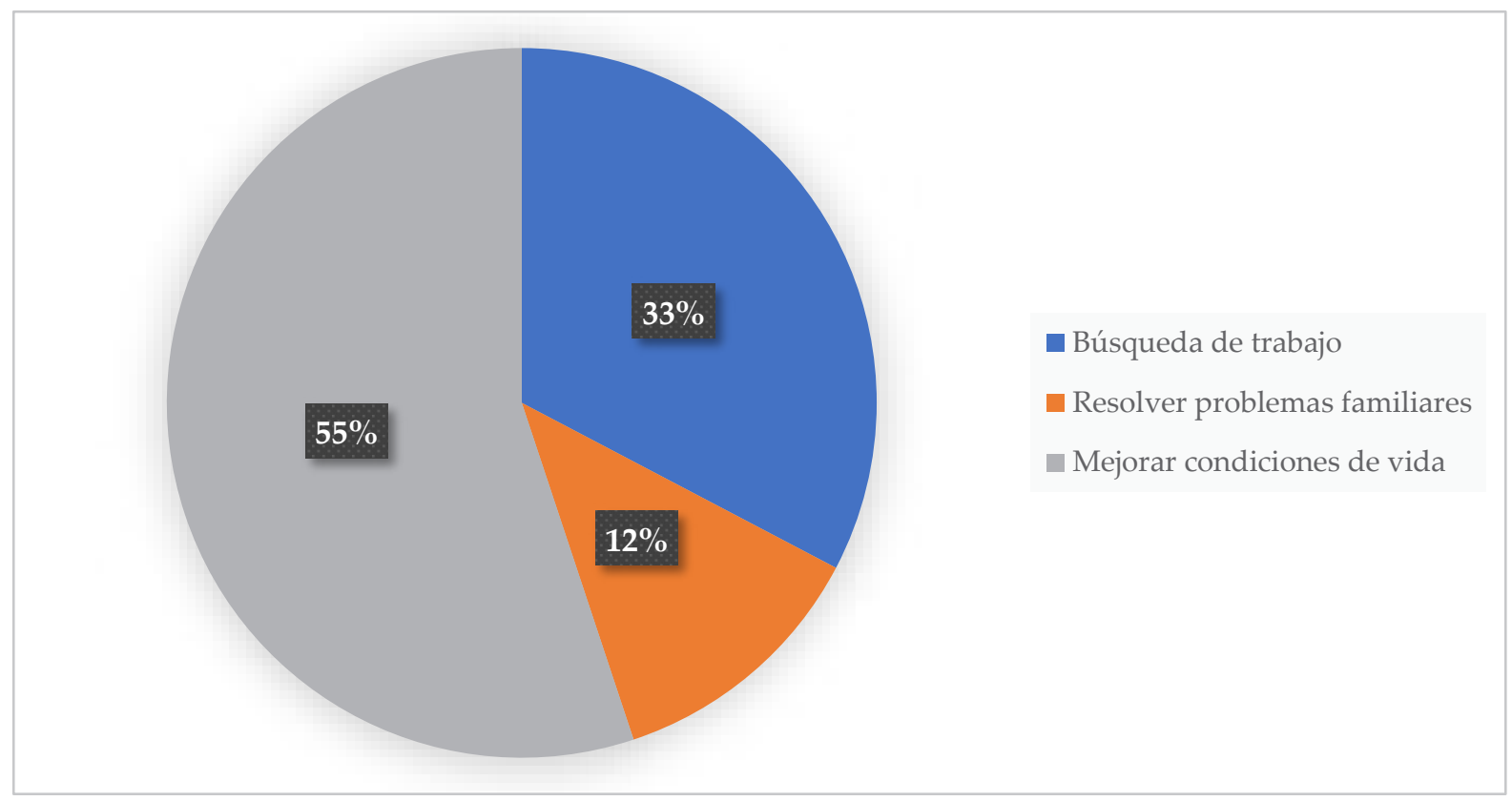

Figura 1. Razones migratorias señaladas por padres de familia y tutores hondureños.

cuado las demandas sociales a sus propios contextos, generando esfuerzos en el tema de la migración escolar. Sin embargo, no se ha visualizado un cambio profundo en las estructuras curriculares, en las metodologías, ni en la formación docente, ya que son evidentes las desigualdades que se presentan en aula escolar. De acuerdo con Pivez Soto (2011, p.140) “en la práctica, las familias inmigrantes viven en barrios segregados, [los niños] acceden a escuelas públicas en deficientes condiciones y experimentan constantemente la discriminación y el racismo". Incluso, existe un grupo específico de adolescentes participantes de procesos migratorios, que son identificados como "Menores Extranjeros No Acompañados (MENAS)"; este grupo social tiene la etiqueta de ser niños de la calle que se encuentran en situación irregular, y de quienes se piensa tienden a aprovecharse de los servicios sociales del Estado de los países de destino.

\section{Procesos migratorios en niños y adolescentes}

La perspectiva de sexo desde la experiencia migratoria en NA hace referencia a las oportunidades, responsabilidades y roles que se asignan por el hecho de ser hombre o mujer. Dentro de los roles que asumen, a partir de los datos etarios de NA deportados, cada vez es más evidente que las edades en que son buscados por los grupos criminales para que participen de forma activa en actos al margen de la ley, como el sicariato, extorsión y tráfico de drogas, entre otros, oscila entre los 9 a 16 años. Al negarse a participar en las estructuras criminales, son amenazados e incluso asesinados, por lo que no tienen otra alternativa que abandonar el país. De acuerdo con 
las Naciones Unidas -División de Migración Internacional (2015) en el apartado de stock de migrantes internacionales por edad y sexo, se indica que la migración de NA en hombres es de 3,230 y 4029 de en mujeres.

\section{Aspiraciones de migración familiar}

Dentro de las aspiraciones de migración familiar más comunes se plantean: mayores ofertas laborales para los padres y mejores oportunidades educativas para los hijos; siendo un fenómeno que se ha marcado contundentemente en las caravanas masivas. Sin embargo, hay algunas características y patrones comunes en la NA migrantes, según la Organización Internacional para las Migraciones, Fondo de Naciones Unidas para la Infancia y Organización Internacional del Trabajo (2013, p.24). Ante esta situación es evidente la falta de políticas públicas específicas que protejan a los NA migrantes, que se encuentran indefensos y endebles; por lo cual se deben generar propuestas de protección y cumplimiento de sus derechos, además de una asistencia que garantice la protección mediante un enfoque diferencial en lo social, biológico y psicológico. Asimismo, en las relaciones según su sexo, edad, origen étnico y su integración en el sector educativo deben primar planes de acción y proyectos como, por ejemplo, establecer una inserción escolar integral que permita atender todas las necesidades que los NA presenten.

Esta propuesta de política constituye un antecedente teórico para la futura toma de decisiones, puesto que los resultados permitirán comprender el protagonismo de las familias hondureñas migrantes o, por lo menos, de los responsables del retorno e integración de los niños y adolescentes. Por último, permitirá valorar hasta dónde la familia migrante cubre o no plenamente su rol representativo en el contexto comunitario y escolar, a través de la contrastación y triangulación de resultados. Estos permitirán en otro momento, hacer una propuesta de Educación reconstructiva para la inserción de NA; determinando un diseño integrador al contexto familiar y escolar, en relación a factores educativos, sociales, culturales y demográficos, a partir de la inserción de políticas educativas y jurídicas. Ello indicará pautas metodológicas flexibles para la mejora del retorno migratorio en el contexto familiar hondureño, articuladas con el sistema educativo formal, no formal e informal.

\section{Metodología}

Para este estudio, se tomaron en cuenta las apreciaciones e interpretaciones de niños pertenecientes a familias migrantes en retorno, de los integrantes de estas familias y de los docentes de centros educativos que las atienden en diversos municipios de cuatro departamentos del país (ver figura 2).

La investigación se ejecutó a partir del enfoque cualitativo con uno de los componentes metodológicos etnográfico. Se realizó una revisión de documentos bajo el método de teoría fundamentada, se utilizaron elementos de tipo descriptivo y diversas variables que permitieron conocer cómo los NA están integrados dentro de sus familias, durante el retorno 


\begin{tabular}{|c|c|}
\hline Departamento & Municipios \\
\hline Francisco Morazán & Distrito Central, Talanga, Cedros y Guaimaca \\
\hline Cortés & Lima, San Pedro Sula y Villanueva \\
\hline Yoro & Morazán, Progreso y Santa Rita \\
\hline Olancho. & Juticalpa \\
\hline
\end{tabular}

Figura 2. Cuadro resumen de departamenos y municipios estudiados.

migratorio. Se desarrollaron tres fases: descriptiva, estructural y protocolaria. A través de las preguntas generadoras comunes a la técnica de la entrevista semiestructurada, se comprendió la experiencia migratoria y las formas de atención del retorno, adquiridas en el contexto familiar comunitario y escolar hondureño.

La aplicación de estas técnicas se inició a partir del planteamiento de las siguientes preguntas generadoras: ¿Qué valoración tiene la experiencia migratoria? ¿Cuáles han sido sus expectativas por migrar? ¿Qué entiende por migrar o huir? ¿Qué condiciones tiene el retorno migratorio? ¿Cuál es su rol en el contexto de familia después del retorno? ¿Se siente integrado en el contexto escolar y comunitario?

En este proceso metodológico se analizó la relación entre la conformación de las familias y el rol representativo en las comunidades, las condiciones y formas de atención a NA en centros educativos de los municipios señalados; la forma de acceso y el consenso con actores clave. Además de las características de los flujos migratorios al interior de los municipios, mediante el monitoreo de estos con estadísticas oficiales. Para ello se aplicaron cuestionarios, entrevistas semiestructuradas y grupos focales a una muestra intencionada con un total de 67 NA, 31 docentes, y 65 personas entre padres y tutores, ubicados en zonas con índices de inseguridad y altos porcentajes de grupos ilícitos en sectores urbano marginal y rural.

Para el desarrollo de la investigación se recurrió a dos formas de recolección de información: presencial y virtual, debido a la crisis sanitaria causada por el COVID-19 y las restricciones nacionales de confinamiento, se implementó una metodología didáctica y lúdica. También se diseñó un manual ilustrativo con indicaciones específicas, dirigido a la estructura de recursos humanos del $\mathrm{Mi}$ nisterio de Educación para fines de culminar la última etapa, y asegurar la recolecta de los audios de las grabaciones. Esto con el fin de triangular e identificar unidades de significados de los informantes, en contraste con las bitácoras recogidas en las primeras visitas en forma presencial. De manera que combina distintas técnicas de investigación cuantitativa y cualitativa para obtener la información de primera mano de los NA migrantes, sus familias y los docentes. 


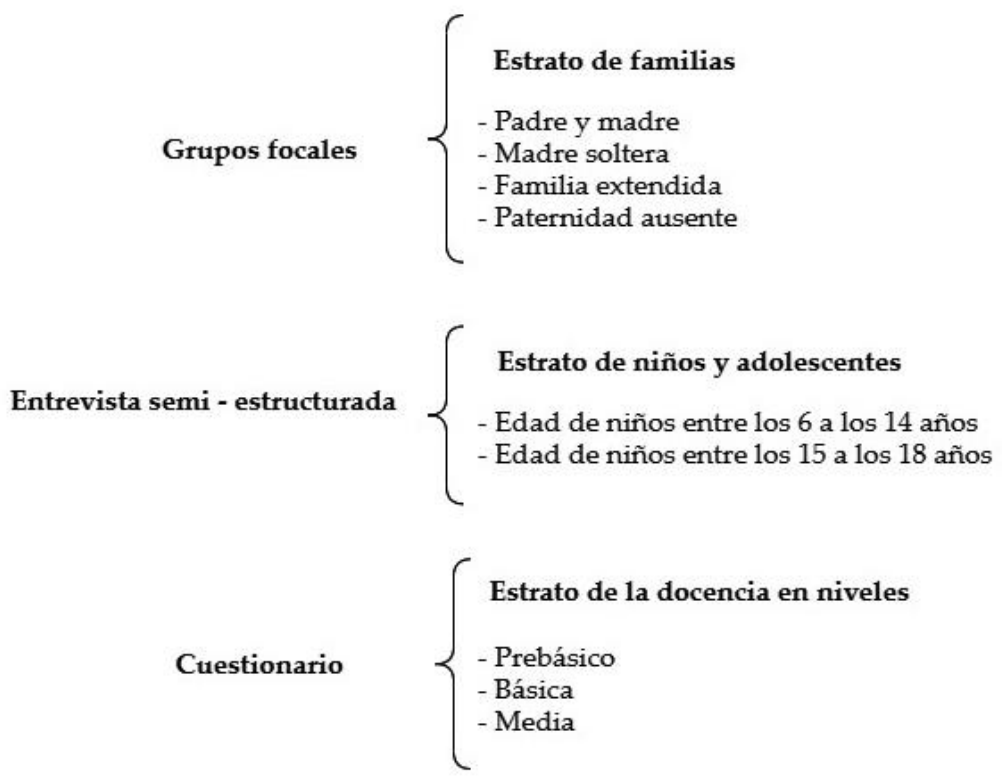

Figura 3. Fases metodológicas del estudio.

El proceso de investigación se dividió en las fases que se desglosan a continuación:

\section{a. Fase 1: Recopilación y sistematización} de información documental disponible sobre el tema y entrevistas a informantes clave. En esta fase se procedió a documentar todos los referentes teóricos sobre el tema de migración en NA, así como datos estadísticos e instituciones nacionales e internacionales que atienen a este sector. De igual manera, se realizó un stakeholding de actores claves, con los cuales se realizaron los primeros enlaces para poder ejecutar la gira de visitas de campo a los departamentos mencionados.

b. Fase 2: Recolección de datos en el campo a NA, familias migrantes y docentes de aula. En esta etapa se visitaron tres de- partamentos: Cortes, Yoro y Olancho. El primero y segundo están ubicados en el norte; y el tercero en el oriente de Honduras. Todos son lugares con reportes de altos porcentajes de familias inmigrantes. Con base en la percepción de los datos obtenidos por las familias hondureñas que emigran del país, el primer motivo de su actuar son los problemas económicos, seguidos de la consecuencia del desempleo; esta categoría se repite en casi todos los estratos, profesionales universitarios $\mathrm{u}$ oficios. Asimismo, se realizó el diseño e instrumentación, validación y pilotaje de estas; aplicación de entrevistas no estructuradas y grupos focales. De esta etapa surgieron alianzas estratégicas con los directores departamentales, apertura para la visita a centros educativos, lazos de 
confianza de padres de familia, niños y jóvenes para entrevistas a profundidad; recolección de información y anécdotas de apoyo para nuevas categorías emergentes.

En el replanteamiento metodológico que surge a partir de la crisis del COVID-19, se concibió un convenio burocrático con la Secretaría de Educación, a través de la Viceministra de Educación en Honduras y su equipo para coordinar con todos los funcionarios, así como el personal del nivel desconcentrado el proceso de investigación, sobre la migración escolar en NA. Esto específicamente con el apoyo y la aplicación de entrevistas y encuestas de forma remota, mediante Whatsapp y videoconferencias en zoom.

Todo este replanteamiento fue un reto para las investigadoras, ya que la situación de confinamiento nacional, hasta la presentación de este artículo, seguía vigente. Sin embargo, la alianza estratégica establecida con las instituciones anteriormente mencionadas, facilitó la comunicación con las familias migrantes identificadas. Es preciso señalar que se elaboró una metodología ilustrada, que se difundió con cada uno de los colaboradores del proceso. Asimismo, se sostuvieron reuniones constantes con el equipo de apoyo técnico que permitió generar como producto una data de material rico y sustantivo, como fue la obtención de grabaciones de las entrevistas con cada familia inmigrante; así como de los niños, adolescentes y personal administrativo de los centros educativos que reportan casos de migración.
Esta fase finaliza con un conversatorio abierto sobre la experiencia de recolección de datos con las familias, convirtiéndose en un hallazgo para este estudio al identificar las formas de relación del rol del docente frente al desafío de la inmigración, de acuerdo con la función laboral. Además del interés e indagación sobre el nivel de conocimiento e involucramiento en atender la integración de NA al contexto escolar.

\section{c. Fase 3: Análisis hacia una política de educación reconstructiva para la toma de decisiones y esquema de propuesta.} Para esta fase fue necesario profundizar sobre la atención de los NA y las tendencias pedagógicas que definen la inclusión social educativa, las cuales confluyen en diferentes formas de inserción del quehacer educativo. En esta investigación, a través de los diversos actores, se indicaron las necesidades y programas requeridos, y se diseñó una propuesta que se pretende entregar a la Secretaría de Educación con el fin de integrarla al aula escolar.

La finalidad de propuesta llamada "Educación Reconstructiva”, postula una oferta educativa para NA, dialógica entre educandos, educadores y comunidad; con patrones culturales articulados a la educación escolarizada, organizada por estructuras académicas, científicas y técnicas. Todo esto con base en lineamientos metodológicos clasificados por campos disciplinares y multidisciplinares, que van desde propuestas curriculares adaptadas a las necesidades académicas de NA, 
programas permanentes y personal capacitado para la atención emocional y psicopedagógica, actividades programáticas recreativas, entre otros.

\section{Resultados}

La educación pública que se ofrece en los diversos países ha demostrado cómo las instituciones escolares se han transformado y adecuado acorde a las demandas sociales y a sus propios contextos, generando esfuerzos en el tema de la migración escolar.

Sin embargo, no se ha visualizado un cambio profundo en las estructuras curriculares, en las metodologías, ni en la formación docente; ya que son evidentes las desigualdades que se presentan en aula escolar. Ante esta situación, uno de los actores educativos indicó:

La verdad es que - quizás - la debilidad es que la Secretaria de Educación no cuenta con una herramienta didáctica para facilitar la atención a los alumnos retornados. Todo recae en el docente o centro educativo. Y no todos contamos con el apoyo de diferentes ONG (Organizaciones no gubernamentales) que atienden esta temática en nuestro país (Entrevista a docente p-12,2020).

Cabe destacar que estas desigualdades en el ámbito escolar hondureño, de acuerdo con lo manifestado por los docentes, se evidencian ante la falta de protocolos y materiales unificados; ya que en las entrevistas manifestaron esta dificultad.
[...] se presenta una dificultad: cuando los niños se van a principio del año [...] cuando ya es mitad del año y el niño retorna, pues no hay problema. En especial, hay problemas en niños de primer grado, porque para reinsertarlos a primero es difícil; mientras que en los demás grados, usted puede avanzar con ellos con clases extras, porque nosotros ya vamos avanzados y no es lo mismo ponerse al día con un niño que ya sabe leer y escribir (Entrevista a docente-p-07,2020).

Asimismo, se reportó un apoyo en el diseño de adaptaciones curriculares en las asignaturas de Matemáticas y Español, por la Comisión Social de Acción Menonita (CSAM); además del apoyo psicológico y espiritual que les brinda a los NA retornados.

En este estudio, el 70.6\% de los NA viven en zonas urbanas y el $29.4 \%$ en zonas rurales. En ambas ubicaciones, los centros educativos a los que asisten se encuentran en zonas de alta vulnerabilidad, pobreza y delincuencia. A todo esto, se agrega que en Honduras se carece de mecanismos de protección estatal para la reinserción; para el caso, el sistema educativo no dispone de proyectos y programas que faciliten el retorno de los NA deportados a las escuelas y colegios. Respecto a los hogares, no hay programas de salud mental que contribuyan a la reinserción de manera saludable, $y$ los centros especializados para garantizar el acompañamiento a la niñez migrante destacan por su ausencia (Casa Alianza Honduras, Pastoral de Movilidad Humana y Catholic Relief Service, 2016, p.54). 
Ante lo expresado por Pavez y Casa Alianza, se denota la necesidad de atención psicológica, social, económica en el contexto escolar comunitario (ver tabla 1).

\section{Condiciones y experiencias migratorias desde la perspectiva de sexo}

La perspectiva de sexo, desde la experiencia migratoria en NA, hace referencia a las oportunidades, responsabilidades y roles que se asignan por el hecho de ser hombre o mujer. Dentro de los roles que asumen, a partir de los datos etarios de NA deportados, cada vez es más evidente que las edades en que son más buscados por los grupos criminales para que participen de forma activa en actos al margen de la ley (como el sicariato, la extorsión y el tráfico de drogas), oscila entre los 9 a los 16 años. Al negarse a participar en las estructuras criminales son amenazados e, incluso, asesinados; por lo que no tienen otra alternativa que abandonar el país. "[...] yo agarré rumbo a los Estados Unidos con mis dos hijas. Tengo tres; pero dejé una con mi papá, pues hay que tener más cuidado con las niñas en esos viajes" (Entrevista a Madre de familia-p-08,2020).

De acuerdo con las Naciones Unidas -División de Migración Internacional (2015), en el apartado de stock de migrantes internacionales por edad y sexo, se indica que la migración de NA en sexo masculino es de 3,230 y 4,029 del sexo femenino.

Las niñas, adolescentes y mujeres migrantes están expuestas a riesgos mayores en cuan- to a discriminación, explotación y violencia, ya sea durante sus travesías o en los lugares de destino. A esto se agrega que "dentro del marco socio-político y económico, la población migrante de países empobrecidos y con características fenotípicas diferentes a las consideradas autóctonas, sufren una sobre exposición en los medios de comunicación y en los ámbitos sociales en los que participan” (Pedone, 2010, p.13).

De esta forma se construyen estereotipos sobre la población migrante, específicamente en la NA que incide en sus vidas cotidianas y en el ámbito del sistema educativo. Ante este panorama, se realizaron hallazgos significativos basados en sexo y edad de la NA, en el cual, los flujos migratorios muestran el cambio de patrones en la convivencia familiar. Estos cambios sociales están provocando en la niñez y la adolescencia una configuración estructural de las familias, surgiendo un tipo de linaje emergente y propia de la globalización como puede ser la familia transnacional. Esta forma mantiene sus vínculos más allá de las fronteras nacionales y se organiza sin perder su sentido de grupo, como por ejemplo las madres que continúan desempeñando su rol en la familia a distancia. 


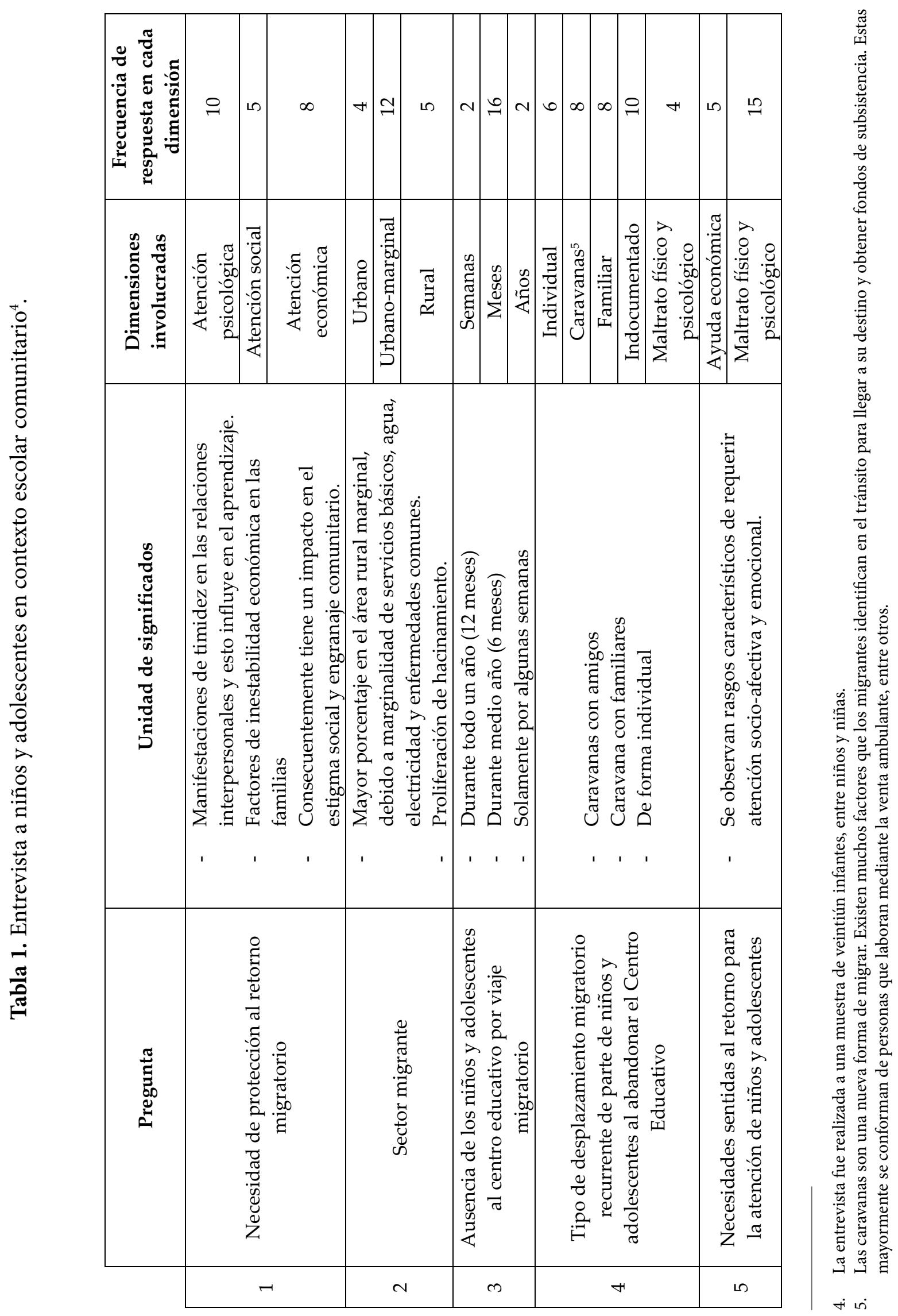




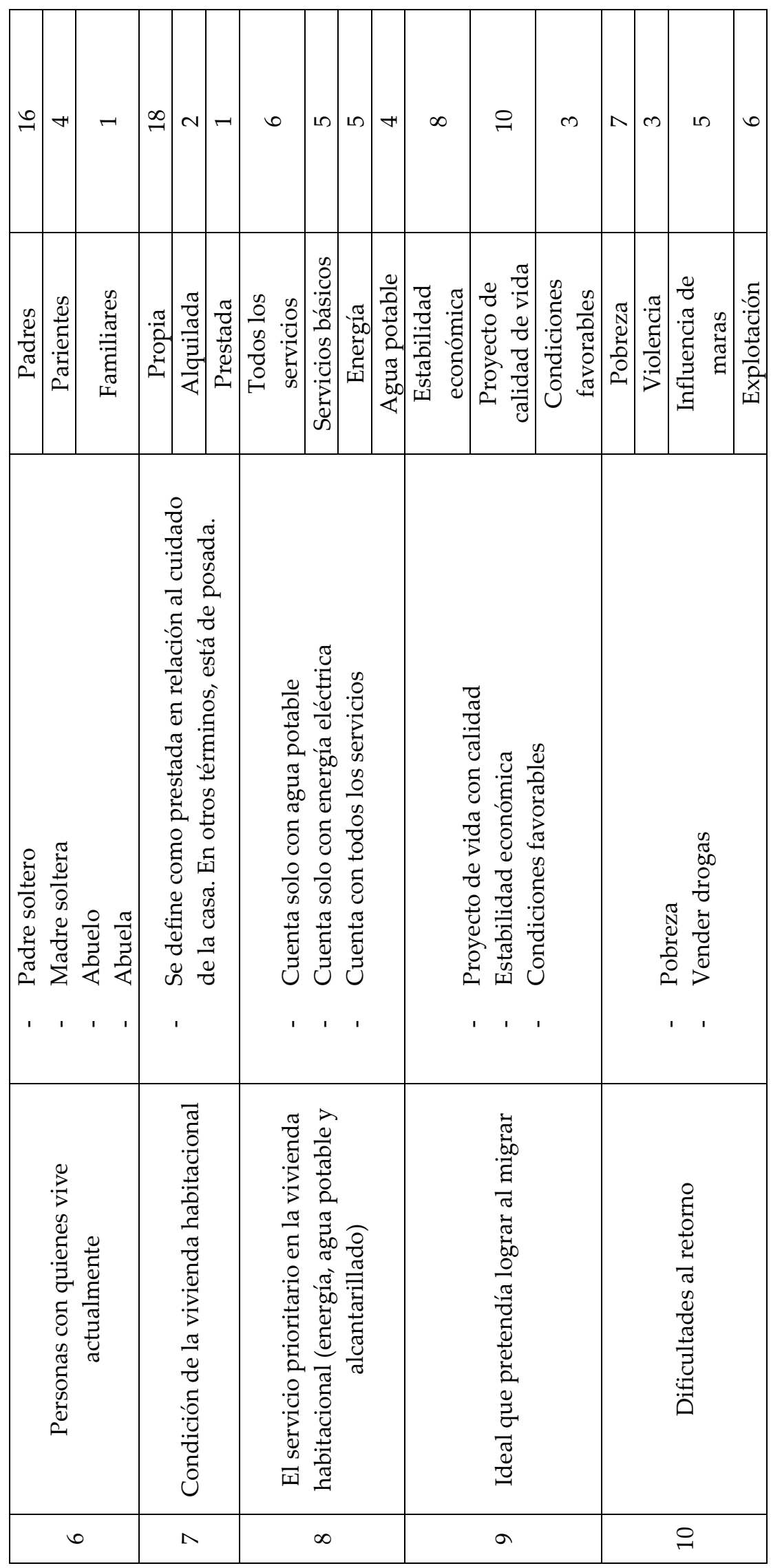


Dentro de las condiciones de experiencias migratorias de los NA, en su retorno del viaje migratorio se encontró que existe un proceso de adaptación para reconocer y recuperar la confianza en sus familiares, ya que el sentimiento de inseguridad vivido sigue siendo latente. Aun así, hay una necesidad e ideal de volver a intentar migrar, y se vuelve un reto para los centros educativos esta retención de estudiantes. Se menciona vehementemente la necesidad de un apoyo psicológico, puesto que, durante el viaje, la mayoría atravesó por experiencias traumáticas de maltrato y visualizaron escenas de muertes durante el proceso migratorio (ver figuras 5 - 8).

\begin{tabular}{|c|c|}
\hline Edades & Categorías consultadas \\
\hline \multirow{10}{*}{$\begin{array}{l}7-10 \\
\text { años }\end{array}$} & Migración forzada e involuntaria \\
\hline & Retorno involuntario \\
\hline & Sentimiento de separación familiar \\
\hline & Miedo por el viaje \\
\hline & Empleo de ambulante \\
\hline & Viaje traumático \\
\hline & Sentimiento de desprotección \\
\hline & Inseguridad \\
\hline & Educación Básica y Media (7º grado como nivel promedio) \\
\hline & Acompañado por sus padres \\
\hline \multirow{7}{*}{$\begin{array}{c}11-14 \\
\text { años }\end{array}$} & $\begin{array}{l}\text { Experiencia vivida al retorno: circularidad migratoria, indocumentados, detención } \\
\text { fronteriza, maltrato físico y psicológico; asilo denegado. }\end{array}$ \\
\hline & Familia extendida \\
\hline & $\begin{array}{l}\text { Ideal de niños y adolescentes: proyecto de vida con calidad, estabilidad económica, } \\
\text { condiciones favorables. }\end{array}$ \\
\hline & $\begin{array}{l}\text { Dificultades a enfrentar cuando retornan: pobreza, vender drogas, violencia entre maras, } \\
\text { explotación infantil, inserción educativa para la niñez del sector rural. }\end{array}$ \\
\hline & Motivación al migrar por amistades y familiares. \\
\hline & Necesidad de apoyo: para ser empático, la parte emocional y tolerancia a los demás. \\
\hline & Dificultades cognitivas en el aprendizaje. \\
\hline
\end{tabular}

Figura 5. Categorización de entrevista a niños y adolescentes retornados entre 7 y 14 años eb contexto escolar comunitario. 


\begin{tabular}{|c|c|}
\hline Categoría & Valoraciones expresadas \\
\hline Niños & $\begin{array}{l}\text { - } \quad \text { Situaciones incómodas en la adaptación de la familia. Porque a veces siento } \\
\text { que estoy con desconocidos. } \\
\text { - } \quad \text { Sentimiento de rencor y no empatía con los demás. Esto me causa estrés. } \\
\text { - } \quad \text { Con deseos de volver a migrar para apoyar a mi familia. } \\
\text { - } \quad \text { Impotencia ante exigencias de grupos ilícitos. } \\
\text { - } \quad \text { Preocupación por quién va a sostener el alimento y enfermedades. } \\
\text { - Necesidad de apoyo en ser empático, la pate emocional y tolerancia con los } \\
\text { demás. }\end{array}$ \\
\hline Niñas & $\begin{array}{ll}\text { - } & \text { Sentimiento de pertenencia al contexto familiar. } \\
\text { - } & \text { Afectación de la convivencia familiar } \\
\text { _ } & \text { Tristeza profunda por el maltrato. } \\
\text { - } & \text { Dificultad en la organización y estructura familiar, acompañados por } \\
\text { momentos de discusión y sentimientos de rencores. } \\
\text { - } \quad \text { Rol para ser madre al cuidado de mis hermanos. } \\
\text { - } \quad \text { Desesperación por la burla en la comunidad, con deseos de no existir. }\end{array}$ \\
\hline
\end{tabular}

Figura 6. Unidades de significado recurrentes entre los niños y niñas (entre 7 a 14 años).

\begin{tabular}{|c|l|}
\hline Edades & \multicolumn{1}{|c|}{ Categorías consultadas } \\
\hline \multirow{4}{*}{$\begin{array}{c}14-18 \\
\text { años }\end{array}$} & Cambios conductuales por viajes migratorios \\
\cline { 2 - 2 } & $\begin{array}{l}\text { Redefinición de roles en contexto comunitario de los integrantes en la familia en aspectos } \\
\text { culturales, sociales e identidad }\end{array}$ \\
\cline { 2 - 2 } & Adecuación curricular por asignaturas y contenidos para la nivelación de aprendizajes \\
\cline { 2 - 2 } & Temporalización de año escolar diferente, asociado al retorno \\
\cline { 2 - 2 } & Preparación psicológica a docentes por estrés \\
\cline { 2 - 2 } & Atención profesional psicológica urgente \\
\cline { 2 - 2 } & Migración interna forzada por amenazas \\
\cline { 2 - 2 } & Reclutamiento de niños y adolescentes por asociaciones ilícitas \\
\cline { 2 - 2 } & Deserción \\
\cline { 2 - 2 } & Ausentismo \\
\hline
\end{tabular}

Figura 7. Categorización de entrevista a niños y adolescentes retornados entre 14 a 18 años en contexto escolar comunitario. 


\begin{tabular}{|l|ll|}
\hline Categoría & \multicolumn{1}{c|}{ Valoraciones expresadas } \\
\hline \multirow{4}{*}{ Adolescentes } & $-\quad$ Existe un potencial enorme para llenar un vacío en materia de atención psico- \\
& afectiva en los adolescentes migrantes en Honduras. \\
& $-\quad$ Se enfrentan problemas con nexos a la migración, tales como la violencia, la \\
& pobreza y el maltrato. \\
& - & Debilidad en la integración de una estrategia enfocada en un retorno protector \\
& más amplio. \\
& La ubicación geográfica de los adolescentes es alterna. \\
& Se observan trastornos emocionales que enfrentan en sus comunidades, lo cual \\
& ha influido en una actitud de mayor sensibilidad y atención hacia estos \\
& problemas. \\
- & Manifiestan desinterés y desesperanza en estudiar. \\
- & Manifiestan atención para la empleabilidad de trabajo. \\
\hline
\end{tabular}

Figura 8. Unidades de significados recurrentes entres los adolescentes (entre 14 a 18 años).

\section{Aspiraciones de migración: ideales y patro- nes culturales}

La emigración se ha producido históricamente por causas diversas. En el caso de los NA en Honduras, las principales causas son la violencia, delincuencia y pobreza. A esto se ahunan factores específicos, directamente ligados al hecho de la colonización, de la descolonización y del crecimiento desigual de las economías estatales y regionales.

Dentro de las aspiraciones de migracion familiar más comunes se plantena: mayores ofertas laborales para los padres y mejores oportunidades educativas para los hijos; fenómeno que se ha marcado contundentemente en las caravanas masivas. Sin embargo, hay algunas características y patrones comunes en los NA migrantes, según la Organización Internacional para las Migraciones, Fondo de Naciones Unidas para la Infancia y Organización Internacional del Trabajo (2013, p.24):

1. Baja escolaridad, por las siguientes razones: falta de recursos, por la lejanía del centro escolar, por la delincuencia en los centros escolares; porque el centro escolar ofrecía educación únicamente hasta sexto grado o porque no les gustaba la escuela.

2. Predominan los hombres, con edades que oscilan entre 8 y 17 años.

3. Incorporación precoz al trabajo, la gran mayoría de las personas menores de edad se habían incorporado al trabajo que pue- 


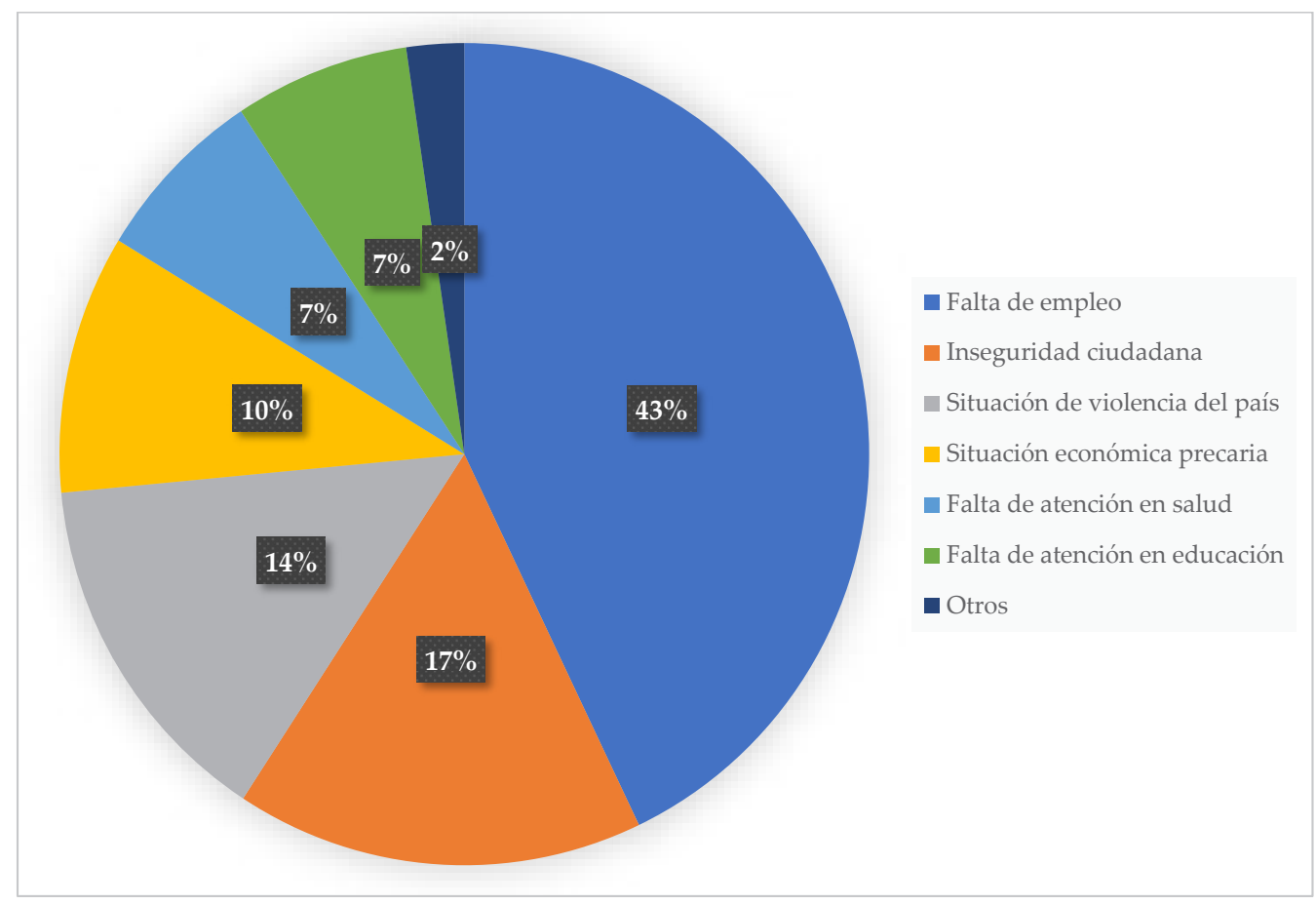

Figura 9. Motivos de migración en padres y tutores.

de ser agrícola, de cuido, de ganado o en construcción, en el caso de los hombres, desde muy tierna edad; y trabajo doméstico en el caso de las mujeres.

4. Provienen de hogares monoparentales, con jefatura femenina.

5. Pobreza y marginalidad, donde los ingresos de las familias provienen del trabajo agrícola, complementado en algunos casos de las remesas que reciben de los padres migrantes. Algunos hogares, inclusive, reciben el bono proveniente del programa social destinado a familias en situación de extrema pobreza.

De acuerdo con este estudio, los ideales de migración en NA se refuerza y visualizan un futuro esperanzador, al emprender el viaje migratorio para la búsqueda de mejores condiciones de vida (ver figura 9).

Sin embargo, las actividades laborales que realizan los niños y adolescentes migrantes transfronterizos son, por lo general, marginales; y se concentran en

las áreas de agricultura y ganadería, sector informal (ventas ambulantes, limpiadores de zapatos, de parabrisas, etc.) y trabajo doméstico. Algunos son utilizados para cometer delitos como tráfico de mercancías o son víctimas de trata de personas con fines de explotación sexual, laboral, etc. (Organización Internacional para las Migraciones, Fondo de Naciones Unidas para la Infancia, \& Organización Internacional del Trabajo, 2013, p.29). 
Por lo que, al regresar o reinsentarse al aula escolar, requieren de un abordaje psicológico y un acompañamiento pedagógico que apoye esta situación. Todo lo anterior, debido al trabajo que ejercen sus padres, y se ven expuestos a reproducir estos patrones de trabajo.

\section{Discusión}

El retorno de la niñez migrante y familias al contexto escolar y comunitario en Honduras debería encaminarse al desarrollo del rol representativo del docente y la familia migrante para la construcción y reproducción de aspectos socio educativos en las comunidades; puesto que están expuestos en la vulnerabilidad de sus derechos, por situaciones tales como bajos recursos económicos, situación de pobreza, separación familiar, marginalidad social, problemas de culturalidad, interrupción de estudios, promoción de la discriminación al retorno, desesperanza, y problemas para la integración al aula y el aprendizaje. Estos aspectos inciden en los altos porcentajes de deserción escolar y ausentismo, producto de la alta intensidad migratoria que a su vez - promueve la exclusión social, con evidencias en las rutas migratorias, denominadas "Caravanas de migración colectiva" y "Migración forzada" para el caso de NA.

Se sugiere que la fase de inserción y tratamiento psicológico del NA debería también acompañarse de momentos de intercambio y trabajo colaborativo, entre los actores, para la participación activa. Por otro lado, en las familias migrantes debería promoverse y fortalecer el rol que representan en sus comunidades; es decir, se debe gestar un proceso de educación reconstructiva, con acciones a la integración del concepto de familia.

Asimismo, se observa el creciente rol de la mujer como proveedora del sostén familiar que se evidencia en las caravanas; también se delega la forma y estilos de crianza en los abuelos. De manera que la representación familiar comunitaria de NA está entre padres jóvenes, en contextos deficitarios por falta de oportunidades y falta de acceso a la educación, además de que la familia se extiende al plano transnacional.

Finalmente, la adecuación del currículo en el contexto migratorio debe generar reflexiones para la toma de decisiones en la construcción de una política por parte del Estado. Esto se propone como educación reconstructiva. Esta, a través de sus representantes, deviene de las acciones de participación colectiva, capacidades de las organizaciones o instituciones; la apertura de la planificación y administración del currículum, la ejecución operativa y el sustento jurídico constitucional que le otorga legitimidad a sus acciones. Todo ello en beneficio de la mayoría, en miras a una política social basada en las relaciones causales de la migración para generar un bienestar común.

Este estudio no podría haberse realizado sin la colaboración del doctor Tomás Botempo, miembro del Consejo de Investigación de Ciencias Sociales CLACSO, con sede en Argentina; de la dra. Claudia Pedone, asesora metodológica, así como de los enlaces de migración de cada departamento de este estudio: maestra Eivy Zelaya, directora del Programa Nacional de Atención Educativa de NA Migrantes Retornados; y maestra Reina 
Rodríguez, enlace de la Dirección Departamental de Cortés. Asimismo, a todas las personas que participaron en los municipios del distrito central, Talanga, Cedros y Guaimaca en el departamento de Francisco Morazán; en los municipios de Lima, San Pedro Sula y Villanueva en el departamento de Cortés; en los municipios de Morazán, Progreso y Santa Rita en el departamento de Yoro y en el municipio de Juticalpa en el departamento de Olancho; quienes de manera directa e indirecta fueron fundamentales en la realización del mismo.

\section{Referencias}

Canales, A. I.; Fuentes-Knight, J. A. y De-León-Escribano, C. R. (2019). Desarrollo y migración: Desafíos y oportunidades en los países del norte de Centroamérica. Comisión Económica para América Latina y el Caribe. https://www.cepal.org/es/publicaciones/44649-desarrollo-migracion-desafios-oportunidades-paises-norte-centroamerica

Casa Alianza Honduras, Pastoral de Movilidad Humana y Catholic Relief Services (2016). Niñas y niños migrantes: Factores de expulsión y desafíos para su reinserción en Honduras. https://imumi.org/documentos/Ninas-y-ninos-migrantes-factores-de-expulsion-y-desafios-para-su-reinsercion-en-Honduras.pdf

Córdova, Y. (2 de agosto de 2018). En Honduras, 26 niños al día dejan la escuela por migrar a Estados Unidos. El Heraldo. https://www.elheraldo.hn/pais/1203342-466/en-honduras26-niños-al-día-dejan-la-escuela-por-migrar-a

Gobierno de la República de Honduras - Centro Nacional de Información del Sector Social (2011 - 2019). Sistema Integral de Atención al Migrante Retornado. http://ceniss.gob.hn/ consultas-1.html

Gobierno de la República de Honduras - Centro Nacional de Información del Sector Social (2020). Objetivos del CENISS. http://www.ceniss.gob.hn/objetivos.html

Gobierno de México (2017). Dirección de investigación. investigación. https://www.incmnsz. $\mathrm{mx} /$ opencms/contenido/investigacion/

Martínez, J. y Fernández, M. (2006). Inmigración y exclusión social. https://www.fuhem.es/ wp-content/uploads/2019/08/MART\%C3\%8FNEZ-Julio-Luis-Inmigraci\%C3\%B3n-yexclusi\%C3\%B3n-social.pdf 
Ministerio de Educación - Guatemala. (2009). Guía de adecuaciones curriculares para estudiantes con necesidades educativas especiales. https://www.mineduc.gob.gt/DIGEESP/documents/Manual_de_Adecuaciones_Curriculares.pdf

Muñoz, T. G. (2003). El cuestionario como instrumento de investigación/evaluación. http://www. univsantana.com/sociologia/El_Cuestionario.pdf

Musalo, K.; Frydman, L. y Ceriani-Cernadas, P. (2015). Niñez y migración en Centro y Norte América: Causas, políticas, prácticas y desafíos. Center for gender \& Refugee studies - Programa Migración y Asilo / Centro de Justicia y Derechos Humanos. https://www.acnur. org/fileadmin/Documentos/Publicaciones/2015/9927.pdf

Observatorio Consular y Migratorio de Honduras (s. f.). Retornados 2020. https://conmigho. $\mathrm{hn} /$ retornados-2020/

Organización Internacional para las Migraciones (2006). Glosario sobre Migración. https://publications.iom.int/system/files/pdf/iml_7_sp.pdf

Organización Internacional para las Migraciones (2015). Conceptos generales sobre migración y niñez: Un referente para la acción en la protección integral de niños, niñas y adolescentes. http://migracion.iniciativa2025alc.org/download/05COe_Conceptos_Migracion_NinCC83ez.pdf

Organización Internacional para las Migraciones - Fondo de Naciones Unidas para la Infancia - Organización Internacional del Trabajo (2013). Niños, niñas y adolescentes migrantes américa central y México. https://rosanjose.iom.int/site/sites/default/files/ninez_america_ latina.pdf

Organización Internacional para las Migraciones - Organización Internacional del Trabajo Coordinación Educativa y Cultural Centroamérica - Red de Observatorios del Mercado Laboral Centroamérica y República Dominicana - Observatorio Latinoamericano de Censos de Población (2011). Flujos migratorios laborales intrarregionales: Situación actual, retos y oportunidades en Centroamérica y República Dominicana. https://www.ilo.org/ wcmsp5/groups/public/---americas/---ro-lima/---sro-san_jose/documents/publication/ wcms_194005.pdf

Parella, S.; Petroff, A.; Speroni, T. y Piqueras, C. (2019). Sufrimiento social y migraciones de retorno: Una propuesta conceptual. Apuntes: Revista de Ciencias Sociales, 46(84), 37-63. https://doi.org/10.21678/apuntes.84.1013 
Pavez-Soto, I. (2011). "Migración infantil: Rupturas generacionales y de género. Las niñas peruanas en Barcelona y Santiago de Chile" [Tesis de doctorado, Universitat Autónoma de Barcelona]. https://www.tdx.cat/handle/10803/79139\#page $=1$

Pedone, C. (2010). Familias, niños, niñas y jóvenes migrantes. Rompiendo estereotipos En Pedone, C. Más allá de los estereotipos: Desafíos en torno al estudio de las familias migrantes (p. 230). IEPALA.

Pocasangre, H. (14 de enero de 2019). Se prepara en Honduras una nueva caravana migrante. República. https://republica.gt/2019/01/14/caravana-migrante-honduras/

Santos-Ramírzez, L. (2020). Geopolítica de las caravanas centroamericanas. FLACSO-UNAH. https://webcache.googleusercontent.com/search?q=cache:s1NDsXXke8gJ:https://flacso. unah.edu.hn/dmsdocument/10086-geopolitica-de-las-caravanas-pdf $+\& c d=1 \&$ hl=es$419 \& \mathrm{ct}=\mathrm{clnk} \& \mathrm{gl}=\mathrm{hn}$

Secretaría de Educación Pública de México (2009). Glosario Educación Superior. http://dsia. uv.mx/cuestionario911/material_apoyo/glosario\%20911.pdf

United Nations - Department of Economic and Social Affairs (2015). International migrant stock 2015. https://www.un.org/en/development/desa/population/migration/data/estimates2/estimates15.asp

Zuazo-Olaya, N. (2013). Causas de la desintegración familiar y sus consecuencias en el rendimiento escolar y conducta de las alumnas de segundo año de la Institución Educativa Nuestra Señora de Fátima de Piura. [Tesis de maestría, Universidad de Piura]. https://pirhua.udep. edu.pe/bitstream/handle/11042/1818/MAE_EDUC_110.pdf 